\title{
Condições de vida e saúde de famílias rurais no sertão cearense: desafios para Agenda 2030
}

\author{
Life and health conditions of rural families in the hinterlands of \\ Ceará: challenges for the 2030 Agenda
}

Luis Lopes Sombra Neto', Flora Viana Elizeu da Silva', Ana Caroline Mendes Barbosa', Fernando Ferreira Carneiro', Vanira Matos Pessoa'

DOI: $10.1590 / 0103-1104202213210$

RESUMO Este estudo caracteriza condições de vida e situação de saúde das famílias que vivem da agricultura familiar e da pesca artesanal no sertão cearense. Trata-se de pesquisa descritiva quantitativa e delineamento transversal realizada mediante entrevistas com 152 agricultores(as) familiares e/ou pescadores(as) artesanais em Novo Oriente, Ceará, Brasil. A análise estatística foi realizada com frequência absoluta e relativa, média aritmética e desvio-padrão. Observaram-se algumas vulnerabilidades vivenciadas pelas famílias: 19,5\% (n=29) com deficiência no tratamento da água para consumo, 25,7\% (n=39) relataram existência de problemas ambientais, 26,6\% (n=40) apresentando insatisfatória produção familiar, 34,6\% (n=47) com baixa renda familiar, 75\% $(n=114)$ com inexistência na localidade de escolas e 88,2\% ( $n=134)$ de creches, $51,3 \%(n=78)$ com carência de opções de lazer e 37,5\% (n=57) em insegurança alimentar. Na análise da situação de saúde, destaca-se prevalência de doenças crônicas, como cardiovasculares e mentais. Essa realidade é contraditória com as metas propostas pelos Objetivos de Desenvolvimento Sustentável: fome zero; boa saúde e bem-estar; educação de qualidade; água limpa e saneamento; emprego digno e crescimento econômico; redução das desigualdades. Dessa forma, as ações de saúde não podem se desvincular dessas iniquidades, devendo-se buscar uma articulação entre governos, empresas e cidadãos para alcançar as metas da Agenda 2030.

PALAVRAS-CHAVE População rural. Saúde da população rural. Características da família. Estratégia Saúde da Família. Atenção Primária à Saúde.

ABSTRACT This study characterizes the living conditions and health situation of families who live from family farming and artisanal fishing in hinterlands of Ceará. This is a descriptive quantitative research and cross-sectional design conducted through interviews with 152 family farmers and / or artisanal fishermen in Novo Oriente, Ceará, Brazil. Statistical analysis was performed with absolute and relative frequency, arithmetic mean and standard deviation. Some vulnerabilities experienced by the families were observed: $19.5 \%(n=29)$ with a deficiency in the treatment of drinking water, $25.7 \%(n=39)$ reported the existence of environmental problems, $26.6 \%(n=40)$ showing unsatisfactory family production, 34.6\% ( $n=47)$ with low family income, $75 \%(n=114)$ with no schools in the locality and $88.2 \%$ ( $n=134)$ of day care centers, $51.3 \%$ ( $n=78)$ with a lack of leisure options, and $37.5 \%$ (n=57) with food insecurity. Prevalence of chronic diseases, such as cardiovascular and mental, stands out. This reality is contradictory with the goals proposed by the SDG: zero hunger; good health and welfare; quality education; clean water and sanitation; decent employment and economic growth; reduction of inequalities. Thus, health actions cannot be separated from these inequities, and a link between governments, companies, and citizens must be sought to achieve the goals of the 2030 Agenda.

1Fundação Oswaldo Cruz (Fiocruz) - Eusébio (CE), Brasil.

luisneto88@hotmail.com
KEYWORDS Rural population. Health of the rural population. Family characteristics. Family Health Strategy. Primary Health Care. 


\section{Introdução}

No Brasil, nas últimas décadas, por meio do movimento da Reforma Sanitária, surgiram críticas ao modelo biomédico com foco exclusivamente na cura das doenças visando à incorporação de um modelo biopsicossocial em que o conceito de saúde estaria associado ao bem-estar físico e mental atrelado às condições de alimentação, moradia, educação, lazer, transporte, trabalho, produção e renda, como definido na Constituição de 1988. No mesmo período, na América Latina, a medicina social desenvolveu o conceito de determinação social da saúde que preconiza uma análise crítica desses múltiplos fatores de estrutura biológica, do modo de vida, das condições coletivas, estruturais e políticas que interagem em conjunto na construção do processo saúde-doença-cuidado'.

Na busca de diminuir essas iniquidades no processo saúde-doença-cuidado mundial, enfocando a erradicação da pobreza, a proteção do planeta e a garantia de que as pessoas alcancem a paz e a prosperidade, a Organização das Nações Unidas (ONU) propõe, incorporados à Agenda 2030, os Objetivos de Desenvolvimento Sustentável (ODS) que abrangem ações para promoção do desenvolvimento social, econômico e ambiental, sendo compostos de 17 objetivos, 169 metas e 231 indicadores a serem discutidos, articulados e alcançados em conjunto pelos três setores da sociedade: governos, empresas e cidadãos ${ }^{2}$.

Nesse contexto da busca do desenvolvimento sustentável, surge a importância da análise do território em que habita uma população, pois este não se limita às suas determinações geográficas, constituindo, na realidade, um processo dinâmico de fatores biológicos, psicológicos, sociais, históricos, culturais, econômicos, políticos, entre outros. Dessa forma, existe uma ampla relação da forma como se organiza uma sociedade com seu processo saúde-doença-cuidado, demonstrando a importância da análise do território como um sistema em constante transformaçãa $0^{\mathbf{3} 4}$.
A avaliação situacional de saúde baseada nos dados relativos ao território adscrito foi incorporada na Estratégia Saúde da Família (ESF), a qual foi criada em 1994, sendo o estado do Ceará pioneiro na sua estruturação. Essa estratégia, um dos pilares da Atenção Primária à Saúde (APS) no Sistema Único de Saúde (SUS), surgiu com o intuito de transformar o modelo anteriormente vigente no sistema público de saúde brasileiro - que era voltado para o assistencialismo individual - em um cuidado em saúde que englobasse promoção, prevenção, vigilância, cura e reabilitação de toda a família. Ao longo dos anos, essa estratégia culminou em melhores indicadores de saúde, como redução da mortalidade infantil, diminuição da incidência de doenças infectocontagiosas, maior cobertura vacinal, diminuição de complicações em gestação, parto e puerpério, aumento do acompanhamento de hipertensos e diabéticos, crescimento do acesso da população à saúde, entre outros ${ }^{5,6}$.

Pelas novas recomendações publicadas na Política Nacional de Atenção Básica ${ }^{7}$, o território adscrito vinculado aos cuidados de cada equipe da ESF deve conter entre $2.000 \mathrm{e}$ 3.500 pessoas, com a média de 2,9 pessoas por família ${ }^{8}$, chegando em torno de 690 a 1.207 famílias. Apesar dessas recomendações, existem algumas realidades, como as vivenciadas pela população rural, que necessita de cuidados mais complexo das equipes da ESF no intuito de analisar a situação de saúde dessas populações, executar ações articuladas de proteção, promoção, vigilância e recuperação da saúde, prevenir riscos e agravos associados às condições de vida, bem como reduzir as vulnerabilidades 9 .

Dentro desse contexto de família como foco das intervenções em saúde, é importante destacar o conceito de família como uma estrutura de relações caracterizadas por influência recíproca, direta, intensa e duradoura entre seus membros. Dessa forma, é fundamental a análise de aspectos como demografia, papéis familiares, moradia, parentesco, transmissão de bens, ciclo vital da família e rituais 
de passagem para uma caracterização mais fidedigna dessas famílias para uma melhor abordagem dos cuidados de saúde. Além disso, as famílias atuais apresentam diversas configurações diferentes do modelo arcaico simplista de pai e mãe biológicos com seus filhos, por exemplo, casais sem filhos, casais homoafetivos, casais com filhos adotivos, avós com os netos, grupos de pessoas não consanguíneas que moram juntas, entre outras ${ }^{10}$.

A alta prevalência dos problemas de saúde encontrados nas famílias associados aos aspectos biopsicossociais é uma importante questão de saúde pública. Um exemplo é o trabalho no meio rural, o qual, muitas vezes, estrutura-se na forma de produção familiar, facilmente presenciada na agricultura familiar e na pesca artesanal, que acaba impactando no modo de vida dessas famílias, que são expostas a diversos fatores de risco de natureza física (acidentes), química (exposição a agrotóxicos, minerais ou metais pesados), biológica (dores osteomusculares, parasitoses), mecânica e ergonômica e psicossocial (transtornos mentais e dependência química) ${ }^{11}$.

Sendo assim, pesquisas sobre famílias que vivem em áreas vulneráveis, como o território rural, precisam serem realizadas para reconhecer as necessidades e particularidades desse grupo com o intuito de reduzir suas fragilidades e minimizar os impactos dos fatores biopsicossociais na construção do processo saúde-doença ${ }^{12,13}$.

Diante dessa reflexão, surge a questão motivadora desta pesquisa: como se apresentam as condições de vida e saúde das famílias que vivem da agricultura familiar camponesa e da pesca artesanal em territórios rurais no sertão do Ceará?

\section{Material e métodos}

Trata-se de estudo descritivo com abordagem quantitativa e delineamento transversal proveniente de entrevistas com agricultores(as) familiares e pescadores(as) artesanais do município de Novo Oriente, classificado pelo Instituto Brasileiro de Geografia e Estatística (IBGE) ${ }^{\mathbf{1 4}}$ como rural adjacente, localizado no estado brasileiro do Ceará.

Foram selecionados para o presente estudo: indivíduos pertencentes à faixa etária entre 18 e 64 anos; economicamente ativos, com atividade produtiva à agricultura familiar ou à pesca artesanal, ou ambas as atividades; residentes em território do sertão/campo há mais de dois anos; integrantes de família adscrita a uma equipe de saúde da família rural. Foi utilizado critério de exclusão: trabalhadores que estão atualmente afastados de suas atividades laborais. Além disso, devido à pandemia ocasionada pelo novo coronavírus no ano de 2020, foram acrescentados aos critérios de exclusão: indivíduos com sintomas de síndrome gripal ou com aferição de temperatura corporal maior ou igual a $37,8^{\circ} \mathrm{C}$.

A amostra deste estudo foi constituída por 152 agricultores/pescadores rurais. A escolha dos participantes da pesquisa ocorreu mediante indicação dos movimentos populares que reconheceram que aquela família é agricultora familiar ou pescadora artesanal, sendo mobilizados pelos líderes comunitários, considerando os critérios de inclusão e exclusão.

A coleta de dados ocorreu durante os meses de setembro e outubro de 2020, por meio da aplicação de formulário elaborado após oficina de 8 horas, com representantes de movimentos populares do campo e das águas, profissionais de saúde do SUS e pesquisadores. Para este estudo, foram utilizadas as variáveis vinculadas às perguntas objetivas do formulário sobre as características biopsicossociais: moradia, ambiente, trabalho, renda, educação, lazer, transporte e alimentação; e histórico familiar de doenças, internações e óbitos das famílias que vivem da agricultura familiar e pesca artesanal.

Posteriormente, o formulário passou por teste-piloto inicial, sendo realizada a sua aplicação por duas pesquisadoras com quatro indivíduos: dois homens e duas mulheres. Em seguida, baseando-se nas demandas 
vivenciadas pelas pesquisadoras do teste-piloto, o formulário passou por processos de revisão.

Para a padronização do método de coleta de dados, os entrevistadores foram treinados, e foi realizado estudo-piloto em agosto de $2020 \mathrm{com}$ amostra não probabilística intencional de 31 trabalhadores rurais do município de Fortim, Ceará, Brasil, que possui dados sociodemográficos semelhantes a Novo Oriente, Ceará; além de ambos serem classificados como rural adjacente ${ }^{\mathbf{1 4}}$.

No momento da entrevista, foram utilizados dispositivos móveis programados com máscara contendo o formulário no aplicativo Open Data Kit (ODK). Os dados inseridos no ODK geram automaticamente uma tabela em plataforma de armazenamento que foi acompanhada virtualmente pelos pesquisadores durante todo o período de coleta. Essa tabela com todos esses dados coletados foi inserida no programa de estatística Statistical Package for the Social Sciences (SPSS) para realização de análise estatística descritiva com estimação da frequência absoluta e relativa, da média aritmética e do desvio-padrão das variáveis.

Este estudo foi desenvolvido respeitando todos os aspectos éticos contidos nas resoluções do Conselho Nacional de Saúde ${ }^{\circ}$ $446 / 2012$ e n $^{0} 510 / 2016$. O projeto de pesquisa foi submetido e aprovado pelo Comitê de Ética em Pesquisa da Escola de Saúde Pública do Ceará com número do Parecer 3.372.478.

\section{Resultados}

O perfil dos entrevistados foi a maioria do sexo masculino com $57,2 \%(n=87)$, predominância na faixa etária entre 51-64 anos com 36,2\% $(n=55)$, sendo a média de $43( \pm 13,2)$ anos. A situação conjugal com maior prevalência foi casado/união estável relatada por $85,5 \%$ $(n=130)$. Em relação à etnia, a maioria deles se autodeclarou como pardos, representado por $67,1 \%(n=102)$; seguido de brancos, $24,3 \%$ $(n=37)$, e negros, $5,3 \%(n=8)$. Sobre a religião, a maioria dos agricultores/pescadores é pertencente ao catolicismo com 82,9\% ( $\mathrm{n}=126)$.

Para responder à pergunta do estudo 'como se apresentam as condições de vida e saúde das famílias que vivem da agricultura familiar camponesa e da pesca artesanal em territórios rurais no sertão do Ceará?' -, foi analisado o conjunto de variáveis sobre as condições de vida e saúde apresentadas pelas famílias residentes em comunidades rurais de Novo Oriente, Ceará, Brasil. Tais variáveis foram agrupadas de acordo com os aspectos biopsicossociais: moradia, ambiente, trabalho, renda, educação, lazer, transporte e alimentação. Conforme descritas na tabela 1.

Tabela 1. Condições biopsicossociais de famílias que trabalham na agricultura familiar camponesa e na pesca artesanal em Novo Oriente, Ceará, Brasil, 2020

\begin{tabular}{lrr}
\hline Variáveis & $\mathbf{n}$ & $\%$ \\
\hline MORADIA & \\
\hline Tipo de casa ( $\mathbf{n}=\mathbf{1 5 2})$ & 151 & 99,3 \\
$\quad$ Casa de alvenaria/tijolo & 1 & 0,7 \\
Outros & 138 & 90,8 \\
Forma de aquisição ( $\mathbf{n}=152)$ & 14,2 \\
Própria-quitada & 9,2 \\
Outra & $3,3( \pm 1,2)$ \\
Número de pessoas que moram na casa $(\mathbf{n}=\mathbf{1 5 2})$ & \\
$\quad$ Média (desvio-padrão) & \\
\hline
\end{tabular}


Tabela 1. (cont.)

\begin{tabular}{|c|c|c|}
\hline Variáveis & $\mathrm{n}$ & $\%$ \\
\hline \multicolumn{3}{|c|}{ Número de pessoas por cômodo no domicílio $(n=152)$} \\
\hline De 1 a 2 pessoas & 122 & 80,3 \\
\hline De 2 a 3 pessoas & 26 & 17,1 \\
\hline 4 ou mais pessoas & 4 & 2,6 \\
\hline \multicolumn{3}{|l|}{ Distância da sede ( $n=117)$} \\
\hline Média (desvio-padrão) km & \multicolumn{2}{|c|}{$14,2( \pm 7,4)$} \\
\hline \multicolumn{3}{|l|}{ AMBIENTE } \\
\hline \multicolumn{3}{|l|}{ Energia elétrica $(n=152)$} \\
\hline Sim & 152 & 100 \\
\hline \multicolumn{3}{|l|}{ Fontes de água para consumo $(n=152)$} \\
\hline Cisterna de placa & 131 & 86,2 \\
\hline Outros & 21 & 13,8 \\
\hline \multicolumn{3}{|l|}{ Tratamento da água $(n=149)$} \\
\hline Filtrada & 70 & 47 \\
\hline Tratada com cloro & 17 & 11,4 \\
\hline Água mineral (comprada) & 5 & 3,3 \\
\hline Mais de uma técnica & 28 & 18,8 \\
\hline Não trata & 29 & 19,5 \\
\hline \multicolumn{3}{|l|}{ Água encanada $(n=152)$} \\
\hline Sim & 149 & 98 \\
\hline Não & 3 & 2 \\
\hline \multicolumn{3}{|l|}{ Avaliação da qualidade da água ( $n=152)$} \\
\hline Ótima & 39 & 25,7 \\
\hline Boa & 111 & 73 \\
\hline Ruim & 2 & 1,3 \\
\hline \multicolumn{3}{|l|}{ Destino das fezes $(n=152)$} \\
\hline Fossa & 141 & 92,7 \\
\hline Mato/quintal & 7 & 4,5 \\
\hline Outros & 4 & 2,8 \\
\hline \multicolumn{3}{|c|}{ Existem problemas ambientais na comunidade $(n=152)$} \\
\hline $\operatorname{Sim}$ & 39 & 25,7 \\
\hline Não & 113 & 74,3 \\
\hline \multicolumn{3}{|l|}{ TRABALHO } \\
\hline \multicolumn{3}{|l|}{ Tipo de atividade laboral $(n=152)$} \\
\hline Agricultura Familiar & 26 & 17,11 \\
\hline Pesca artesanal & 38 & 25 \\
\hline Agricultura e pesca & 19 & 12,5 \\
\hline Agricultura e trabalho doméstico & 42 & 27,63 \\
\hline Pesca e trabalho doméstico & 25 & 16,45 \\
\hline Agricultura, pesca e trabalho doméstico & 2 & 1,32 \\
\hline
\end{tabular}


Tabela 1. (cont.)

\begin{tabular}{|c|c|c|}
\hline Variáveis & $\mathbf{n}$ & $\%$ \\
\hline \multicolumn{3}{|c|}{ Destino da pesca ou plantio $(n=152)$} \\
\hline Consumo familiar & 22 & 14,5 \\
\hline Venda & 3 & 2 \\
\hline Consumo familiar e venda & 127 & 83,5 \\
\hline \multicolumn{3}{|c|}{ Como a família identifica seu trabalho e produtos $(n=152)$} \\
\hline Trabalho e produção individual & 12 & 8 \\
\hline Regime de economia familiar & 139 & 91,4 \\
\hline Regime de parceria & 1 & 0,6 \\
\hline \multicolumn{3}{|c|}{ Avaliação da produção nos últimos 6 meses $(n=152)$} \\
\hline Ruim & 5 & 3,3 \\
\hline Regular & 35 & 23 \\
\hline Boa & 95 & 62,5 \\
\hline Ótima & 17 & 11.2 \\
\hline \multicolumn{3}{|l|}{ RENDA } \\
\hline \multicolumn{3}{|l|}{ Fontes de renda da família $(n=152)$} \\
\hline Somente pesca artesanal & 3 & 2 \\
\hline Somente benefício do governo & 6 & 4 \\
\hline Benefício do governo e outros & 109 & 71,7 \\
\hline Criação animais e outros & 23 & 15,1 \\
\hline Outros & 11 & 7,2 \\
\hline \multicolumn{3}{|c|}{ Valor de renda mensal familiar $(n=136)$} \\
\hline Até $R \$ 500$ & 47 & 34,6 \\
\hline$R \$ 501-1.000$ & 45 & 33 \\
\hline$R \$ 1.001-1.500$ & 25 & 18,4 \\
\hline$R \$ 1.501-2.000$ & 19 & 14 \\
\hline
\end{tabular}

\section{EDUCAC̣ÃO}

Escola na comunidade $(n=152)$

Sim

$38 \quad 25$

Não

$114 \quad 75$

Creche para criança até 3 anos $(n=152)$

$\begin{array}{lrr}\text { Sim } & 18 & 11,8 \\ \text { Não } & 134 & 88,2\end{array}$

Distância da moradia para escola/creche $(n=110)$

Média (desvio-padrão)

Existe transporte escolar $(n=152)$

\begin{tabular}{lrr} 
Sim & 149 & 98 \\
Não & 3 & 2 \\
\hline LAZER & & \\
\hline
\end{tabular}

Existem opções de lazer na comunidade $(n=152)$

\begin{tabular}{llr} 
Sim & 78 & 51,3 \\
Não & 74 & 48,7 \\
\hline
\end{tabular}


Tabela 1. (cont.)

\begin{tabular}{lrr}
\hline Variáveis & $\mathbf{n}$ & $\%$ \\
\hline TRANSPORTE & \\
\hline Família possui transporte próprio (n=152) & 141,8 \\
Sim & 11,2 \\
Não & 99 \\
Tipo de transporte (n=152) & 65,1 \\
Motocicleta & 4,6 \\
Outros (automóvel e bicicleta) & 23 \\
Mais de um transporte & 35 \\
Sem transporte próprio & 11 \\
\hline ALIMENTAC̣ÃO & 7,3 \\
\hline Preocupação com alimentos acabarem na casa nos últimos 12 meses (n=152) & \\
Sim & 57 \\
Não & 37,5 \\
Deixou de comer alimentos favoritos por falta de produção/dinheiro nos últimos 12 meses (n=152) & 62,5 \\
Sim & 47 \\
Não & 105 \\
Limitou variedade de alimentos por falta de produção/dinheiro nos últimos 12 meses (n=152) & 31 \\
Sim & 69 \\
Não & 40 \\
\hline
\end{tabular}

Fonte: elaboração própria, 2021

n: amostra absoluta; \%: frequência relativa.

Como observado na tabela, as famílias possuem condições de moradia com casas do tipo alvenaria/tijolo em 99,3\% ( $\mathrm{n}=151)$, e forma de aquisição própria-quitada em 90,8\% $(n=138)$. A média de pessoas morando na casa foi de $3,3( \pm 1,2)$, culminando na maioria das moradias com 1 a 2 pessoas por cômodo em 80,3\% ( $\mathrm{n}=122)$. A distância da casa à sede do município obteve média de $14,2 \mathrm{~km}( \pm 7,4)$.

Sobre as condições do ambiente em que vivem as famílias de agricultores(as) familiares e pescadores(as) artesanais, os achados foram: todos os domicílios possuem energia elétrica, a principal fonte para consumo da água utilizada pela família é a cisterna de placa em $86,2 \%$ ( $n=131)$ e o tratamento ocorre por meio de filtração em $47 \%(n=70)$; entretanto, percentual significativo de $19,5 \%(n=29)$ referiu que não realiza tratamento da água para consumo. Além disso, praticamente todas as famílias possuem água encanada relatado por $98 \%$ ( $n=149)$, e avaliam a qualidade da água como boa com $73 \%(n=111)$. No que se refere ao destino das fezes, $92,7 \%(n=141)$ referiram que ocorre principalmente em fossas. Além disso, 25,7\% ( $\mathrm{n}=39)$ dos indivíduos descreveram existir problemas ambientais na comunidade.

Em relação ao trabalho, além das atividades laborais da agricultura familiar e da pesca artesanal, houve grande prevalência do trabalho doméstico associados ao trabalho na agricultura e/ou pesca com 45,4\% ( $\mathrm{n}=69)$. O destino da pesca ou plantio é principalmente para venda e consumo familiar em $83,5 \%(n=127)$. A maioria das famílias identifica seu trabalho como regime de economia familiar com $91,4 \%$ ( $n=139)$, e avaliação da produção nos últimos 6 meses como 'boa' referida por $62,5 \%(n=95)$, apesar de porcentagem significativa de $26,6 \%$ $(n=40)$ ter avaliado como 'regular' ou 'ruim'. 
Nas questões referentes à renda, verifica-se que mais de $70 \%$ (n=109) das famílias obtêm sua renda proveniente de benefício do governo e outras fontes (agricultura e/ou pesca e/ou criação de animais). $\mathrm{O}$ valor da renda familiar destacou-se a menor faixa de valores equivalente a até $\mathrm{R} \$ 500$ em 34,6\% (n=47).

Sobre a educação, foi descrito que não existem escolas por $75 \%(n=114)$ ou creches por $88,2 \%(n=134)$ na comunidade. Apesar disso, a distância da moradia teve média de 6,5 $\mathrm{km}( \pm 5,6)$, e existe transporte escolar para os alunos em $98 \%(n=149)$. Em relação ao lazer, foi relatado por mais da metade dos entrevistados que não existia opções de lazer na comunidade com $51,3 \%(n=78)$ no momento da pesquisa. Dados satisfatórios foram observados sobre o transporte, em que 92,8\% (n=141) das famílias alegam possuir transporte próprio, sendo o tipo principal a motocicleta com $65,1 \%(n=99)$.

Alguns dados preocupantes foram descritos sobre a alimentação, em que quantidade significativa de agricultores(as) familiares e pescadores(as) artesanais descreveram nos últimos 12 meses: preocupação com alimentos acabarem em 37,5\% ( $n=57)$, deixou de comer alimentos favoritos por falta de produção/ dinheiro em $31 \%(n=47)$, e limitou variedade de alimentos por falta de produção/dinheiro em $26,3 \%(n=40)$.

Nas variáveis relacionadas com as condições de saúde, foram analisados os dados sobre problemas de saúde, internações e óbitos das famílias, que vivem da agricultura familiar e da pesca artesanal, os quais foram coletadas, durante as entrevistas, por meio da citação individual, pelo entrevistador, das causas mais comuns de acordo com o perfil brasileiro ${ }^{15,16}$ para que o entrevistado optasse pela existência ou inexistência de casos na família.

As variáveis relacionadas com os problemas de saúde já apresentados na família foram agrupadas, no intuito de facilitar a análise, nos grupos: infectocontagiosas, crônicas não transmissíveis, psicossociais, complicações de causas preveníveis e causas externas; assim como se observa na tabela 2 .

Tabela 2. Problemas de saúde já apresentados, por ordem de frequência, nas famílias que trabalham na agricultura familiar camponesa e na pesca artesanal em Novo Oriente, Ceará, Brasil, 2020. ( $n=152)$

\begin{tabular}{lrr}
\hline PROBLEMAS DE SAÚDE & $\mathbf{n}$ & $\%$ \\
\hline INFECTOCONTAGIOSOS & 128 & 84,2 \\
\hline Catapora & 86 & 56,5 \\
Sarampo, caxumba ou rubéola & 39 & 25,6 \\
Dengue & 28 & 18,4 \\
Coronavírus - Covid-19 & 23 & 15,1 \\
Chikungunya & 16 & 10,5 \\
Tuberculose & 6 & 3,9 \\
Hanseníase & 5 & 3,2 \\
Infecção sexualmente transmissível & 2 & 1,3 \\
Zika & & 74,3 \\
\hline BIOLÓGICOS NÃO TRANSMISSívEIS & 113 & 57,8 \\
\hline Hipertensão arterial & 88 & 52,6 \\
Refluxo/gastrite & 80 & 48,6 \\
Diabetes mellitus & 74 & \\
Asma & & 73 \\
\hline
\end{tabular}


Tabela 2. (cont.)

\begin{tabular}{|c|c|c|}
\hline PROBLEMAS DE SAÚDE & $\mathrm{n}$ & $\%$ \\
\hline Neoplasia (câncer) & 67 & 44 \\
\hline Acamado/Domiciliado & 50 & 32,8 \\
\hline Deficiente físico & 34 & 22,3 \\
\hline Epilepsia & 33 & 21,7 \\
\hline Obesidade & 32 & 21 \\
\hline Desnutrição & 24 & 15,7 \\
\hline \multicolumn{3}{|l|}{ PSICOSSOCIAIS } \\
\hline Ansiedade & 75 & 49,3 \\
\hline Depressão & 63 & 41,4 \\
\hline Demência/Alzheimer & 35 & 23 \\
\hline Usuário de drogas & 34 & 22,3 \\
\hline Autismo/deficit cognitivo & 13 & 8,5 \\
\hline \multicolumn{3}{|c|}{ COMPLICAC̣ÕES DE CAUSAS PREVENÍVEIS } \\
\hline Acidente vascular encefálico & 57 & 37,5 \\
\hline Infarto agudo do miocárdio & 53 & 34,8 \\
\hline \multicolumn{3}{|l|}{ CAUSAS EXTERNAS } \\
\hline Acidente de moto & 49 & 32,2 \\
\hline
\end{tabular}

Fonte: elaboração própria.

n: amostra absoluta de famílias; \%: frequência relativa de famílias.

Nos problemas de saúde já apresentados nas famílias de agricultores(as) familiares e pescadores(as) artesanais, encontraram-se maiores prevalências nos grupos de problemas de saúde infectocontagiosos, destacando-se catapora em 84,2\% (n=128) e sarampo/caxumba/ rubéola em $56,5 \%(n=86)$, e biológicos não transmissíveis, principalmente hipertensão arterial em $74,3 \%$ ( $n=113)$, doenças do refluxo gastroesofágico em $57,8 \%(n=88)$ e diabetes mellitus em 52,6\% $(n=80)$.

Também é importante destacar o número expressivo de casos no grupo de problemas psicossociais, como ansiedade em $49,3 \%(n=75)$ e depressão em $41,4 \%(n=63)$. Além disso as complicações de causas preveníveis: acidente vascular encefálico em $37,5 \%(n=57)$ e infarto agudo do miocárdio em $34,8 \%(n=53)$.

As internações e os óbitos ocorridos nas famílias nos últimos cinco anos estão descritos na tabela 3, demonstrando uma prevalência em ordem decrescente: hipertensão com $27,6 \%(n=42)$, acidente automobilístico com $21 \%(n=32)$ e problemas cardíacos com 20,3\% $(\mathrm{n}=31)$. As três primeiras causas de óbitos referidos, em ordem decrescente, foram: problemas cardíacos com 19,7\% ( $\mathrm{n}=30)$, neoplasia com $15,3 \%(n=23)$ e acidente automobilístico com $11,8 \%(n=18)$. 
Tabela 3. Casos de internações e/ou óbitos, por ordem alfabética, nos últimos cinco anos, nas famílias que trabalham na agricultura familiar camponesa e da pesca artesanal de Novo Oriente, Ceará, Brasil, 2020. ( $n=152)$

\begin{tabular}{lrr}
\hline & INTERNAC̣̃̃ES & ÓBITOS \\
PROBLEMAS DE SAÚDE & $\mathbf{n}(\%)$ & $\mathbf{n}(\%)$ \\
\hline Acidente de trânsito & $32(21)$ & $6(11,8)$ \\
Covid-19 & $13(8,5)$ & $2(1,3)$ \\
Diarreia & $30(19,7)$ & $2(1,3)$ \\
Doença mental & $10(6,5)$ & $3(1,9)$ \\
Hipertensão & $42(27,6)$ & $23(15,1)$ \\
Neoplasia & $28(18,4)$ & $4(2,6)$ \\
Pneumonia & $15(9,8)$ & $30(19,7)$ \\
Problemas cardíacos & $31(20,3)$ & $1(0,7)$ \\
Tuberculose & $4(2,6)$ & \\
\hline
\end{tabular}

Fonte: elaboração própria.

n: amostra absoluta de famílias; \%: frequência relativa de famílias.

O agravo por problemas cardíacos, diferentemente dos outros analisados, apresentou praticamente o mesmo número de internações com 20,3\% ( $n=31)$ e de óbitos com 19,7\% ( $n=30)$, que pode estar associado a maior gravidade desses casos, que poderiam ser detectados precocemente e acompanhados na APS.

\section{Discussão}

Os achados demonstram vulnerabilidades biopsicossociais experimentadas pelas famílias que vivem da agricultura familiar e/ou pesca artesanal no território rural, como: preocupação com a alimentação (insegurança alimentar), deficiência no tratamento da água para consumo, existência de problemas ambientais, insatisfatória produção familiar, baixa renda familiar, inexistência de escolas ou creches na comunidade, carência de opções de lazer na comunidade. Essa realidade é contraditória com as metas propostas pelos ODS: fome zero; boa saúde e bem-estar; educação de qualidade; água limpa e saneamento; emprego digno e crescimento econômico; redução das desigualdades; combate às alterações climáticas, demonstrando uma necessidade de restruturação urgente para enquadrar-se nas perspectivas da Agenda 2030 para o Desenvolvimento Sustentável2.

Esses problemas biopsicossociais interferem direta ou indiretamente nas condições de vida e saúde dessas famílias. Dessa forma, essa realidade acarreta dados comprovadamente piores em indivíduos que moram nessas áreas comparados aos que moram em área urbana, como autoavaliação de saúde regular/ruim/ muito ruim (45\%/32\%), conectado à rede coletora de esgoto ou pluvial (5\%/65\%), sem água canalizada no domicílio (21\%/1\%), lixo coletado diariamente (27\%/92\%), e há lugar público para atividade física $(11 \% / 48 \%)^{17}$.

Em pesquisa realizada em comunidades ribeirinhas da Amazônia, ao aplicar índice de salubridade ambiental domiciliar para o meio rural, foi constado que um dos principais fatores que contribuem para insalubridade e baixa salubridade é a precariedade dos domicílios em relação à existência de instalação sanitária domiciliar, a qual inviabiliza o acesso à água dentro da casa e, também, ao sabão para práticas de higiene, além da coleta e do tratamento de esgotos entre outras atividades relacionadas com a saúde ambiental18. Apesar de dados satisfatórios descritos neste estudo relacionados com a salubridade ambiental, um dado instigante é sobre o número expressivo de 
famílias que não tratam a água para consumo, sendo a principal fonte da água para consumo proveniente de cisternas de placas. Esse fato pode relacionar-se ao número expressivo, em pleno século XXI, de causas diarreicas nas internações hospitalares.

Em análise técnica de água de fontes rurais usadas para abastecimento domiciliar e na agroindústria ${ }^{19}$, verificaram-se: cor da água abaixo do padrão máximo permitido; baixa concentração de amônia; $\mathrm{pH}$ com variável entre 5,5 e 7,5 e algumas amostras com contaminação por coliformes fecais. Além disso, apesar de o Brasil ser um país rico em recursos hídricos, são notórias as desigualdades relacionadas com o acesso à água, refletindo contrastes no desenvolvimento econômico e social. Em estudos relativos à distribuição espacial dos indicadores operacionais do serviço de abastecimento de água na região Nordeste, é possível identificar problemas referentes ao acesso, consumo e perdas de água, inclusive com índice de consumo de água com percentuais considerados elevados em determinadas localidades dos estados da Bahia, do Ceará, de Alagoas e da Paraíba ${ }^{20}$.

Alguns dados encontrados mostraram-se satisfatórios, como tipo de casa, forma de aquisição da moradia, energia elétrica, fontes de água para consumo por meio de cisternas de placas e destino das fezes em fossas. Uma das explicações para essa realidade pode ser em virtude da atuação de alguns programas governamentais, como Bolsa Família, Luz para Todos, Um Milhão de Cisternas e Saneamento Brasil Rural, que proporcionam melhorias nas condições de vida dessas famílias. Apesar dos benefícios comprovados desses programas governamentais, muitos deles estão enfrentando, desde 2016, uma escassez gradativa nos recursos financeiros destinados.

A produção proveniente do trabalho rural descrita como 'regular', 'ruim' ou 'muito ruim' por número significativo de entrevistados, além de interferir na renda familiar, também pode gerar consequências na alimentação da família que utiliza, muitas vezes, parte da produção para consumo, configurando-se como regime de economia familiar em que o trabalho mútuo dos membros da família é indispensável à própria subsistência e ao desenvolvimento socioeconômico da família ${ }^{21}$. Essa realidade foi observada neste estudo, utilizando itens adaptados da Escala Brasileira de Insegurança Alimentar (Ebia) ao encontrar números significativos associados à preocupação e à limitação da alimentação das famílias que vivem da agricultura familiar e/ou pesca artesanal ${ }^{22}$.

Esses dados convergem com outros estudos realizados com aplicação da escala Ebia na população cearense que destaca que um percentual de $44,2 \%$ dos domicílios se encontrava na condição de insegurança alimentar no estado, sendo pior nas áreas rurais (53,4\%) do que nas urbanas (41,7\%). Esse fato demonstra a importância da agricultura familiar no combate à insegurança alimentar, pois atua desde a produção até a formação de mercado e agroecologia. Dessa forma, ações promovidas por programas governamentais, como Programa de Aquisição de Alimentos (PAA) e Programa Nacional de Alimentação Escolar (PNAE), são exemplos que devem ser expandidos, uma vez que incentivam compras da agricultura familiar para abastecer os equipamentos públicos e entidades da rede socioassistencial ${ }^{23}$.

Um dos motivos que podem ter agravado essa insegurança alimentar, detectado pelo estudo, é devido à pandemia da Covid-19 no período da coleta de dados. Os efeitos dessa crise na saúde tornam-se ainda mais graves no trabalho informal e na incerteza de acesso à renda e às políticas públicas como no contexto do Nordeste. Essa realidade envolve questões que vão além da oferta ou do acesso ao alimento ou da geração de renda, demandando estratégias que contribuam para a efetivação do conjunto dos direitos sociais e em uma proteção social que atenda a todas as necessidades básicas do ser humano, principalmente neste período atual que se vivencia ${ }^{23,24}$.

Esse tema torna-se ainda mais importante no contexto atual vivido pela sociedade 
brasileira, que presencia o desmonte de políticas ambientais, de segurança e soberania alimentar e bem-estar social, contradizendo direitos fundamentais propostos pela ONU, como alimentação adequada ${ }^{2}$. Dessa forma, o fortalecimento dos sistemas agroalimentares sustentáveis é fundamental para criação de política públicas que se articulem com a promoção de saúde e segurança alimentar e nutricional25.

Todos esses fatores contribuem para problemas de saúde, internações e óbitos nos membros pertencentes a famílias rurais. $\mathrm{Na}$ análise da saúde realizada neste estudo, identificaram-se dados semelhantes aos encontrados nos últimos anos no Brasil, em que, diante da sua transição epidemiológica, observa-se uma tendência na diminuição das doenças infectocontagiosas e crescimento de doenças crônicas, como cardiovasculares e mentais. Também foram encontrados números expressivos de óbitos por doenças cardiovasculares, neoplasias e causas externas, semelhantes às principais causas de mortalidade brasileira ${ }^{15,16}$.

É interessante observar os dados relativos aos casos de Covid-19 nas famílias analisadas na pesquisa, pois eles foram coletados aproximadamente seis meses após a confirmação dos primeiros casos no Ceará, iniciando-se os diagnósticos na capital do estado. A relação dinâmica existente entre os territórios urbanos e rurais, principalmente nessas áreas classificadas como rurais adjacentes como Novo Oriente (CE), provoca um contato físico constante entre os sujeitos. Além disso, algumas vulnerabilidades, como a pobreza, as desigualdades socioeconômicas e as altas taxas de trabalho informal, são fatores responsáveis pela intensificação no número de $\operatorname{casos}^{26}$.

Outro dado da pesquisa que merece destaque é sobre a alta prevalência de problemas, internações e óbitos associados às causas externas, destacando-se os acidentes. Uma das explicações para esse fato pode ser em razão da quantidade expressiva de indivíduos que possuem como transporte próprio a motocicleta no município. Dessa forma, é fundamental a realizações de ações educativas na prevenção desses agravos.

Sobre dados de internações hospitalares no Brasil, o IBGE ${ }^{27}$ caracteriza que 6,6\% (13,7 milhões) de brasileiros entrevistados ficaram internados em hospitais por 24 horas ou mais nos 12 meses, sendo que 64,6\% (8,9 milhões) realizaram esse atendimento por meio do SUS, inclusive com proporções ainda mais expressivas na região Nordeste (77,8\%). Além disso, no meio rural, as internações em serviços públicos também foram mais frequentes $(85,9 \%)$ do que as registradas na área urbana (61,4\%).

A ESF, que possui o seu foco de atuação na abordagem familiar, está diretamente relacionada com a promoção e a prevenção desses problemas de saúde no intuito de diminuir esses casos de internações e óbitos. Dessa forma, a ESF deve estar preparada para lidar com as singularidades do processo de adoecimento de cada grupo e as especificidades nos seus aspectos biopsicossociais, bem como articular a vigilância em saúde com as ações em promoção, prevenção, tratamento e reabilitação de maneira adequada e eficaz ${ }^{\mathbf{1 3}}$.

Entretanto, ainda existe uma heterogeneidade na consolidação da APS no Brasil em função, principalmente, dos diferentes processos de gestão dos municípios, que possuem autonomia para definir as prioridades para os gastos em saúde, podendo decidir os investimentos que serão vinculados ou não à APS. Essa priorização nos gastos destinados à saúde pública e aos cuidados biopsicossociais é de extrema importância diante da realidade atual de austeridade fiscal do governo que ameaça a expansão e a consolidação do SUS. Na medida em que o fortalecimento da APS é uma das mais potentes medidas nos cuidados em saúde, a diminuição de investimentos na APS representa o enfraquecimento da consolidação de arranjos sistêmicos, capazes de garantir a coordenação e a integralidade da atenção, 5 ,28,29.

Diante dessa realidade, emerge a importância da Política Nacional de Saúde Integral das Populações do Campo, da Floresta e das Águas (PNSIPCFA) no intuito de trazer maior 
visibilidade para essas desigualdades vivenciadas por esse segmento populacional na busca por melhores indicadores das famílias que vivem da agricultura e da pesca, por meio de um adequado acesso aos serviços de saúde de qualidade para reduzir os riscos associados aos aspectos biopsicossociais ${ }^{30}$.

\section{Conclusões}

Assim como caracterizado no presente estudo, existem muitas vulnerabilidades biopsicossociais que podem contribuir para as condições de vida e de saúde das famílias que vivem da agricultura familiar e/ou pesca artesanal. Por isso, as ações de saúde pública não podem se desvincular dessas condições biopsicossociais associadas às iniquidades em saúde, devendo buscar uma articulação entre governos, empresas e cidadãos no intuito de alcançar as metas dos ODS.

Além disso, os serviços de saúde devem realizar o planejamento de suas ações com base na análise situacional dos problemas de saúde. Destaca-se neste estudo que a insegurança alimentar e as doenças crônicas, como doenças cardiovasculares e problemas de saúde mental, são questões prioritárias para serem enfrentadas pelo SUS e por outras políticas intersetoriais como as relacionadas com a Agenda 2030. A ESF nesses territórios pode ter um importante papel na abordagem desses problemas por meio da articulação com outras políticas intersetoriais e com sociedade civil organizada visando a sua superação mediante iniciativas e ações territoriais. $\mathrm{O}$ fomento à convivência com o semiárido e a agroecologia é uma importante estratégia há muito tempo desenvolvida por organizações - como a Articulação Semiárido Brasileiro (ASA) - que poderiam estar mais articuladas ao SUS e à ESF.

Diante das peculiaridades de vida e saúde nos territórios rurais, bem como dos desafios colocados para os profissionais de saúde atuantes nessas comunidades, os quais precisam compreender as dimensões do modo de vida e trabalho rural, com seus riscos, é fundamental visibilizar o que se passa no campo, já que a maioria dos estudos no Brasil se concentra nas populações urbanas. Portanto, mais pesquisas necessitam serem realizadas no intuito de melhorar a abordagem das famílias que vivem e sobrevivem do meio rural, bem como aprofundar o conhecimento sobre os territórios e os modos de produzir saúde. Hoje se vive a maior tragédia humanitária do século com a pandemia da Covid-19, que vulnerabiliza ainda mais as populações sertanejas e faz de agendas para a vida, como a Agenda 2030, um guia para a ação imediata envolvendo governos e sociedade civil organizada.

\section{Colaboradores}

Sombra Neto LL (0000-0002-0204-1960)*, Silva FVE (0000-0002-6053-3189)*, Barbosa ACM (0000-0002-7591-6799)*, Carneiro FF (0000-0002-6625-9715)* e Pessoa VM (00000003-3676-9607)* contribuíram igualmente para a elaboração do manuscrito. 


\section{Referências}

1. Borghi CMSO, Oliveira RM, Sevalho G. Determinação ou determinantes sociais da saúde: texto e contexto na América Latina. Trab. Educ. Saúde. 2018;16(3):86997.

2. Organização das Nações Unidas. Objetivos de Desenvolvimento Sustentável (ODS). Nova York: ONU; 2015. [acesso em 2021 jan 28]. Disponível em: https:// brasil.un.org/pt-br/sdgs.

3. Pessoa VM, Almeida MM, Carneiro FF. Como garantir o direito à saúde para as populações do campo, da floresta e das águas no Brasil? Saúde debate. 2018; 42(esp1):302-314.

4. Souza S, Pappen M, Krug SBF, et al. Uma revisão narrativa associando a vulnerabilidade à saúde e os fatores ambientais de trabalhadores rurais. Rev. Bras. Med. Trab. 2018; 16(4):503-508.

5. Neves RG, Flores TR, Duro SMS, et al. Tendência temporal da cobertura da Estratégia Saúde da Família no Brasil, regiões e Unidades da Federação, 2006-2016. Epidem. Serv. Saúde. 2018 [acesso em 2021 jan 28]; 27(3). Disponível em: http://www. scielo.br/scielo.php?script=sci_arttext\&pid=S2237$-96222018000300302 \& \operatorname{lng}=$ pt\&nrm=iso\&tlng=pt.

6. Macinko J, Mendonça CS. Estratégia Saúde da Família, um forte modelo de Atenção Primária à Saúde que traz resultados. Saúde debate. 2018; 42(esp1):18-37.

7. Brasil. Ministério da Saúde. Portaria $n^{\circ} 2.436$, de 21 de setembro de 2017. Aprova a Política Nacional de Atenção Básica, estabelecendo a revisão de diretrizes para a organização da Atenção Básica, no âmbito do Sistema Único de Saúde (SUS). Diário Oficial da União. 22 Set 2017.

8. Instituto Brasileiro de Geografia e Estatística. Coordenação de Geografia. Pesquisa Nacional por Amostra de Domicílios contínua (PNAD contínua). [acesso em 2021 jan 28]. Disponível em: https://www.ibge. gov.br/estatisticas/sociais/habitacao.html.
9. Carneiro FF, Pessoa VM, Teixeira ACA, organizadores. Campo, floresta e águas: práticas e saberes em saúde. Brasília: Editora UnB; 2017. [acesso em 2021 jan 28]. Disponível em: http://books.scielo.org/id/ wnvqt.

10. Pereira Neto ÉF, Ramos MZ, Silveira EMC. Configurações familiares e implicações para o trabalho em saúde da criança em nível hospitalar. Physis. 2016; 26(3):961-979.

11. Dantas CMB, Dimenstein M, Leite JF, et al. Território e determinação social da saúde mental em contextos rurais: cuidado integral às populações do campo. ATHENEAD. 2020; 20(1):2169.

12. Junqueira FM, Melo Cabral ER, Carneiro FF, et. al. A pesquisa brasileira em contextos rurais: instrumento de justiça social. In: Savassi LCM, Almeida MM, Floss M, et al., organizadores. Saúde no caminho da roça. Rio de Janeiro: Editora Fiocruz; 2018. 163 p. (Coleção Fazer saúde).

13. Lima MC, Teixeira ACA, Barros E, et al. O fazer rural. In: Savassi LCM, Almeida MM, Floss M, et al., organizadores. Saúde no caminho da roça. Rio de Janeiro: Editora Fiocruz; 2018. 163 p. (Coleção Fazer saúde).

14. Instituto Brasileiro de Geografia e Estatística. Coordenação de Geografia. Classificação e caracterização dos espaços rurais e urbanos do Brasil: uma primeira aproximação. Rio de Janeiro: IBGE, Instituto Brasileiro de Geografia e Estatística; 2017.

15. Brasil. Ministério da Saúde, Departamento de Informática do SUS. SINAN: Sistema de Informação de Agravos de Notificação. Brasília, DF: Ministério da Saúde; 2020. [acesso em 2020 dez 15]. Disponível em: http://www2.datasus.gov.br/DATASUS/index.php?area $=0203 \&$ id $=29878153$.

16. Brasil. Ministério da Saúde, Departamento de Informática do SUS. SINAN: Sistema de Informação sobre Mortalidade. Brasília, DF: Ministério da Saúde; 2020. 
[acesso em 2020 dez 15]. Disponível em: http://www2. datasus.gov.br/DATASUS/index.php?area=060701.

17. Savassi LCM, Almeida MM, Floss M, et al., organizadores. Apresentação. In: Savassi LCM, Almeida MM, Floss M, et al., organizadores. Saúde no caminho da roça. Rio de Janeiro: Editora Fiocruz; 2018. 163 p. (Coleç ão Fazer saúde).

18. Bernardes C, Bernardes RS, Günther WMR. Proposta de índice de salubridade ambiental domiciliar para comunidades rurais: aspectos conceituais e metodológicos. Eng. Sanit. Ambient. 2018; 23(4):697-706.

19. Sampaio CAP, Ide GM, Batalha CP, et al. Análise técnica de água de fontes rurais. Eng. Sanit. Ambient. 2019; 24(2):213-217.

20. Araújo SC, Silva Filho JA, Silva GMS, et al. Distribuição espacial de indicadores operacionais de serviço de abastecimento de água no Nordeste Brasileiro. Rev. Verde. 2016; 11(1):20.

21. Neri EL, Garcia LG. Atrizes da roça ou trabalhadoras rurais? O teatro e a fachada para obtenção da aposentadoria especial rural. Soc. Estado. 2017; 32(3):701724.

22. Brasil. Ministério do Desenvolvimento Social e Combate à Fome. Escala Brasileira de Insegurança Alimentar - EBIA: análise psicométrica de uma dimensão da Segurança Alimentar e Nutricional. Brasília, DF: Ministério do Desenvolvimento Social e Combate à Fome; 2014.

23. Instituto de Pesquisa e Estratégia Econômica do Ceará. PRAD Informe: Análise da (in)segurança alimentar do Ceará a partir dos dados da Pesquisa Regional por Amostra de Domicílios (PRAD-CE). v. 3. Fortaleza: IPECE; 2021.

24. Schappo S. Fome e insegurança alimentar em tempos de pandemia da covid-19. SER_Social. 2021 [acesso em 2021 jan 28]; 23(48):28-52. Disponível em: https:// periodicos.unb.br/index.php/SER_Social/article/ view/32423.

25. Pereira N, Franceschini S, Priore S. Qualidade dos alimentos segundo o sistema de produção e sua relação com a segurança alimentar e nutricional: revisão sistemática. Saude soc. 2020; 29(4):e200031.

26. Kerr L, Kendall C, Silva AAM, et al. COVID-19 no Nordeste brasileiro: sucessos e limitações nas respostas dos governos dos estados. Ciênc. Saúde Colet. 2020; 25(supl2):4099-4120.

27. Instituto Brasileiro de Geografia e Estatística. Pesquisa Nacional de Saúde. Rio de Janeiro: IBGE; 2019. 181 p. [acesso em 2021 mar 5]. Disponível em: https:// www.ibge.gov.br/estatisticas/sociais/saude/9160-pesquisa-nacional-de-saude.htlm?=\&t=o-que-e.

28. Castro MC, Massuda A, Almeida G, et al. Brazil's unified health system: the first 30 years and prospects for the future. The Lancet. 2019; 394(10195):345-356.

29. Almeida PF, Medina MG, Fausto MCR, et al. Coordenação do cuidado e Atenção Primária à Saúde no Sistema Único de Saúde. Saúde debate. 2018; 42(esp1):244-260.

30. Brasil. Ministério da Saúde. Portaria no 2.311, de 23 de outubro de 2014. Altera a Portaria n ${ }^{\circ} 2.866 / \mathrm{GM} /$ MS, de 2 de dezembro de 2011, que institui, no âmbito do Sistema Único de Saúde (SUS), a Política Nacional de Saúde Integral das Populações do Campo e da Floresta (PNSIPCF). Diário Oficial da União. 24 Out 2014.

Recebido em 18/06/2021

Aprovado em 04/11/2021

Conflito de interesses: inexistente

Suporte financeiro: Programa Inova Fiocruz (Edital Geração de Conhecimento-Novos Talentos, Edital número 3 de 2018) 